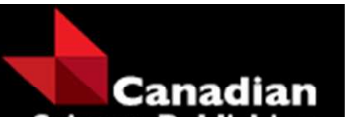

Science Publishing

Canadian Journal of Forest Research Revue canadienne de recherche forestière

\title{
Forest Fibre Network Design with Multiple Assortments: a case study in Newfoundland
}

\begin{tabular}{|c|c|}
\hline Journal: & Canadian Journal of Forest Research \\
\hline Manuscript ID & cjfr-2016-0504.R2 \\
\hline Manuscript Type: & Article \\
\hline Date Submitted by the Author: & $11-J u n-2017$ \\
\hline Complete List of Authors: & $\begin{array}{l}\text { Abasian, Foroogh; Universite Laval, Département de génie } \\
\text { mécanique,Pavillon Adrien-Pouliot, bureau } 3511 \\
\text { Rönnqvist, Mikael; Université Laval, Département de génie mécanique } \\
\text { Ouhimmou, Mustapha; Ecole de technologie superieure }\end{array}$ \\
\hline Keyword: & $\begin{array}{l}\text { Forest biomass, value chain design, backhaul transportation, sorting yards, } \\
\text { integrated strategic and tactical planning }\end{array}$ \\
\hline $\begin{array}{l}\text { Is the invited manuscript for } \\
\text { consideration in a Special } \\
\text { Issue? : }\end{array}$ & N/A \\
\hline
\end{tabular}

\section{SCHOLARONE \\ Manuscripts}


1 Forest Fibre Network Design with Multiple Assortments: a case study in Newfoundland

2 Foroogh Abasian ${ }^{1,3}$, Mikael Rönnqvist ${ }^{1,3}$, Mustapha Ouhimmou ${ }^{2,3}$

$3{ }^{1}$ Université Laval, Department of Mechanical Engineering, Québec (QC), Canada,

$4{ }^{2}$ École de technologie supérieure, Department of Automated Production Engineering, Montréal

5 (QC), Canada,

$6{ }^{3}$ Interuniversity Research Centre on Enterprise Networks, Logistics and Transportation

7 (CIRRELT)

8 Corresponding author: Foroogh Abasian, Foroogh.abasian.1@ulaval.ca 
Abstract

10 The Canadian forest industry is facing several challenges including high fibre cost, decline in

11 commodity profitability and low investment levels at relatively old manufacturing plants. To

12 enable transformation of the industry, innovations are needed to develop value-added products

13 and to shift to an efficient integrated value chain. In this regard, improved logistics for better

14 handling of raw material, forest biomass utilization, and use of new technologies are some

15 promising avenues. In this paper, we propose a generic value chain model which includes

16 locating new sorting yards and biorefineries maximizing the overall profit of the value chain.

17 This integrated planning problem deals with strategic decisions including investments in new

18 facilities and technologies, and tactical decisions comprising backhaul transportation and fibre

19 flows across the value chain. To solve such a problem, we developed a mixed integer

20 programming model to design the forest value chain network. This model is used in an industrial

21 case study in the province of Newfoundland, Canada. We have generated and analyzed 32

22 scenarios evaluated on 12 predefined key performance indicators. The results show that

23 collaboration through backhauling, common terminals, and new assortments are important

24 opportunities to improve the profitability and efficiency of the value chain. The potential

25 improvement over the current situation is as high as $23 \%$ considering the aforementioned 26 actions.

27

28 Key words: Forest biomass, value chain design, backhaul transportation, sorting yards, 29 integrated strategic and tactical planning. 


\section{INTRODUCTION}

31 In recent years, the forest industry crisis has had significant economic and social consequences.

32 Several studies have investigated the causes and effects of these crises on the Canadian forest

33 industry (Mockler and Fairbairn 2009; Mockler and Robichaud 2011). These studies show that

34 the crisis in the industry has been provoked by a rapid decline in commodity demand, the volatile

35 exchange rate of the Canadian dollar as well as strong competition from Asian companies. To

36 deal with the aforementioned challenges, the industry should first stabilize its market conditions,

37 then smoothly transform along four distinct lines: These are market development, operational

38 efficiency, business process change and new product development (Natural Resources of Canada

39 (A) 2016). Among them, new product development, in particular green energy from forest

40 biomass, has been accentuated recently. Such development requires network redesign to

41 introduce new facilities and technologies into the supply chain.

42 It is often more difficult to redesign a network than to design one from start. There is a need to

43 consider the ongoing operations and to ensure smooth transition between the two designs.

44 Moreover, the reengineering process can become even more complex when there are several

45 stakeholders with their own objectives who have run their operations for many years. In reality,

46 this is often the situation that must be dealt with primarily. Hence, our goal is to develop a

47 decision support tool for all transition phases. The tool first proposes improvement directions for

48 current state of supply chain. Afterwards, it provides new network design for new transformed

49 industry.

50 To propose improvement strategies, it is essential to have insights about the entire forest supply

51 chain. D’Amours et al. (2008) presented a global view of Canadian forest supply chain. They

52 characterized the forest supply chain, in particular the Canadian one, as an integrated and 
53 divergent supply chain. The forest wood flow starts with harvesting and then continues through

54 sorting, transportation, storage and operating to convert to products comprised of lumber, pulp

55 and paper, energy or new bio-products (Carlsson and Rönnqvist 2005). All final products are

56 distributed to the market. Wood flow planning consdering interactions between all business units

57 was studied by Weintraub and Epstein (2005).

58 The sorting and transportation parts of wood flow have significant impacts on efficiency and 59 economy of supply chain. Sorting activities help to reduce variability of supply to the mills that 60 require wood supply with specific properties. Such supply strengthens the competitiveness of the 61 industry since it makes possible high-quality products. In this regard, log sorting yards are 62 among the improvement strategies for the forest economy (Alam et al. 2014). Several objectives 63 including profit making, cost saving, risk reduction and an improved forest management 64 economy could be achieved through strategic and operational planning of sorting yards (Dramm 65 et al. 2004). In spite of its advantages, Carlgren et al. (2006) showed, for a case study, the introduction of demand for a specific log assortment will increase the transportation and sorting 67 costs by up to $6 \%$. However, they noted that backhaul transportation could reduce the cost by up 68 to $25 \%$. Hence, backhaul transportation plays a significant role in cost reduction.

69 There are several comprehensive studies on developing decision support systems for 70 transportation planning with special consideration for backhauling (Carlsson and Rönnqvist 71 2007; Forsberg et al. 2005). Effective backhaul routes depend notably on stakeholders' 72 cooperation. Palander and Väätäinen (2005), demonstrated a significant cost saving from 73 backhauling combined with interenterprise collaboration in the Finnish forest industry.

74 Besides determining improvement directions, our tool has been developed to propose a smooth 75 market transformation. This could happen in forest industry by invaluable forest biomass, which 
76 consists of forest residues, non-merchantable woods, sawdust and other non-usable by-products.

77 These homogenous resources have been used for many years to provide forest industry energy.

78 However, with rising environmental concerns, the industry in such resources as a means of

79 producing renewable energies has increased. Frombo et al. (2009) provided a strategic decision

80 model to select an optimal energy-conversion technique that maximizes the selling profit minus

81 collection and transportation costs. Gunnarson et al. (2004) studied tactical planning for forest

82 bioenergy supply chain in which they satisfied the yearly contract-based energy demands. A

83 comprehensive study by Shabani et al. (2013) reviewed all recent researches on forest biomass

84 value chain optimization for bioenergy production.

85 Furthermore, forest residues are a great alternative to produce biofuels and bio-products because

86 of their wide geographical distribution and availability. Ekşioğlu et al. (2009) developed a mixed

87 integer programming (MIP) model for biorefinery supply chain producing ethanol from

88 agriculture and woody biomass. They proposed a network design for biorefinery location

89 coordinated with harvesting, sorting, transporting and processing decisions. Kim et al. (2011)

90 considered a forest supply chain comprising forest, potential location for biofuel plants and

91 market with objective of determining number, location and size of biofuel plants as well as

92 logistic planning. They performed a sensitivity analysis showing the significant effect of demand

93 variation on network design and profitability.

94 Integration of bioenergy and biofuel value chain in forest industry has been studied by Cambero

95 et al. (2014). The authors proposed a multi-period optimization model to decide the right period

96 for facility opening considering procurement planning. However, real integration in supply chain

97 consists in considering all existing partners. Troncoso et al. (2015) compared the integrated

98 forest value chain planning versus decoupled planning for a Chilean case study. The authors 
99 reported that the net present value of value chain increased up to $5 \%$ when the proposed 100 integrated solution is implemented.

101 Feng et al. (2010) clarified the potentiality of integrated forest biomass value chain. The paper 102 proposed a general mathematical programming model to locate new biorefinery, assign 103 technologies to them and allocate resources. New technology could develop in green field sites 104 or existing mills. Dansereau et al. (2012) considered a pulp and paper mill which has facilities 105 for biorefinery products. They considered manufacturing flexibility methodology to reduce the 106 risk of assessing new technologies.

107 In this paper, we followed the concept of an integrated forest supply chain of Feng et al. (2010) 108 in a more comprehensive way. The main decisions we consider are a) to locate value-adding 109 facilities including biorefineries and sorting yards b) to assign technology and capacity to 110 biorefineries c) to allocate resources to existing and new facilities d) to plan the logistics of 111 supply chain considering transportation, sorting and operating and finally f) to consider the 112 possibility of collaboration between industrial partners including backhaul transportation. Our 113 general problem is motivated by a specific case of the forest industry in the province of 114 Newfoundland, Canada. We propose an MIP model to investigate the aforementioned problem. 115 All required information is collected from stakeholders in the province and FPInnovations, a 116 Canadian non-profit organization in forestry research. The results of this project are reported in 117 the Centre for Forest Science and Innovation of Newfoundland to be utilized as a guideline for 118 industry improvement and competitiveness policies. The main contributions of the paper are the 119 proposed model and its practical test in a real industrial case study setting.

120 The outline of the paper is as follows: Section 2 gives the problem description. Then the detailed 121 case study is described in Section 3. Section 4 reports the results based on a set of designed 
122 scenarios. Finally, we provide some concluding remarks.

\section{PROBLEM Formulation AND MATHEMATICAL MODEL}

124 Forest supply chain comprises four main entities: the forest, pulp and paper operation units, 125 wood-manufacturing units to produce lumber, panel and engineering wood, and transformation 126 units (Lehoux et al. 2012). Each of these entities has its own business plan to maximize its 127 revenue and profit; however, they are all interconnected (Figure 1). Manufacturing plants are 128 connected to the forest either directly or through terminals. Sawmills provide intermediate 129 products for pulp and paper mills and transformation mills. Finally, the products are delivered to 130 the market through distribution centres. A short description of each entity is provided in the 131 following.

132 Forest- Canada has the second largest forest in the world with 396 million hectares. There are 35 133 different economic important species. Forests and their industries play a vital role in the 134 Canadian economy, society and environment. They support one million jobs, i.e., $5.6 \%$ of the 135 total employment of the country (Natural Resources of Canada (B) 2016).

136 Terminal- Terminals are in-transit yards for logs from the forest and before their arrival in mills.

137 They can be used as sorting yards, stock-yards or merchandizing yards (Lehoux et al. 2012).

138 Terminals provide a great opportunity for backhaulage for all stakeholders in the network and 139 can extensively increase the existing value of fibre in the network by allocating the right log to 140 the right mill with a minimum cost.

141 Sawmill- Products and by-products of sawmills have a crucial role in the efficiency of the entire 142 forest supply chain. Wood chips produced in the sawing process are utilized as raw material for 143 pulp and paper mills. Moreover, bark, sawdust, and shavings are used as feed for new 144 transformation processes. 
145 Pulp and paper mill- An important part of the industry is the pulp and paper industry. However,

146 the rise of electronic media and the steep decline in paper-based products has affected the main

147 economic contributors of the forest industry. In this regard, their collaboration in the

148 transformation of Canadian forest industry has dramatically increased.

149 Transformation unit- They are new facilities to start industry transformation. Several processes

150 including extraction, combined heat and power, organic ranking cycle, pelletization,

151 fermentation, and lignin precipitation could be employed at these units to produce biofuels,

152 biochemicals, biodegradable plastics, personal care products and industrial chemicals from forest

153 biomass. Among aforementioned processes, pelletization is a mature one with high yield.

154 Moreover, the stable market of pellets, motivates investors towards such a low-risk investment

155 option. In this regard, we consider pelletization as the process of our transformation unit to be 156 included in the value chain network.

\section{$157 \quad 2.1$ Optimization model}

158 We propose an MIP model to find an optimal design for a general forest supply chain 159 considering existing stakeholders, potential terminals and pellet mills, and backhaul availability.

160 The model aims to maximize the profit of the network while satisfying a set of constraints such

161 as customer demand, forest supply and mill capacities, existing road network, etc. The following 162 will present the exact formulation of the MIP model including sets and parameters definition, 163 decision variables and constraints.

164 Defined and supporting sets for the developed model are described in the following.

$165 P^{R} \quad$ set of raw and intermediate products

$166 \quad P^{F} \quad$ set of final products

$167 P \quad$ set of all product types $P=P^{R} \bigcup P^{F}$ 
$168 \quad S \quad$ set of sort groups

$169 \quad F^{P} \quad$ set of piles

$170 \quad F^{C} \quad$ set of species

$171 \quad D \quad$ set of districts

$172 \quad Z \quad$ set of zones

$173 O$ set of tenure holders

$174 \quad L^{T} \quad$ set of potential terminal locations

$175 \quad L^{E} \quad$ set of potential biorefinery locations

$176 \quad M^{P} \quad$ set of existing manufacturing plants

$177 \quad N \quad$ set of all network nodes $N=Z \cup M^{P} \cup L^{T} \cup L^{E}$

$178 \quad R^{i} \quad$ set of existing and candidate processes for mill $i$

$179 \quad H \quad$ set of hauling type

$180 \quad L^{B} \quad$ set of backhaul routes

181 Here are some comments on set of sort groups and set of hauling types; We define four sort

182 groups comprising unsorted supply, sort base on wood species, sort base on wood species and 183 their utilization, i.e., sawlogs, studwoods (short sawlogs), pulpwoods and energy woods and sort 184 base on wood species, utilization and dimension. The model allows sorting at different places 185 from higher sort group to lower ones. Moreover, we classify different truck organization within 186 the set of hauling types.

187 Supporting sets:

$188 I_{i z o} \quad$ set of forest areas indicated by district $i$, zone $z$ and tenure holder $o$. 
$189 H_{o j h} \quad$ set of hauling methods for woods belonging to $o$ with truck type $h$ to mill $j$.

$190 \quad P_{r j}^{R} \quad$ set of all processes $r$ at mill $j$.

$191 P_{p r}^{R+} \quad$ set of input products $p$ to process $r$.

$192 P_{p r}^{R-} \quad$ set of output products $p$ from process $r$.

$193 W_{l f}^{F S} \quad$ set of sorted pile $f$ created from sorting pile $l$.

$194 E_{f o j}^{F F} \quad$ flow of pile $f$ from forest tenure $o$ to mill $j$.

$195 E_{\text {foij }}^{F M}$ flow of pile $f$ of tenure holder $o$ from mills $i$ to $j$.

196 The parameters used to describe harvest areas, costs, values, production at mills, and capacities

197 are given below.

$198 k_{i z o p}$ annual allowable cut of product $p$ at forest block indicated by $(i z o) \in I_{i z o}$.

$199 a_{f l}^{F S}$ proportion of sorted pile $f$ at pile $l$.

$200 \quad f_{i} \quad$ fixed cost to open new facility $i$.

$201 \quad v_{p j}^{F} \quad$ unit selling value of final product $p$ at the gate of mill $j$.

$202 \quad c_{S}^{I} \quad$ unit harvesting cost for each sort group $S$.

$203 \quad c_{s j}^{S} \quad$ unit sorting cost to group $S$ at mill $j$.

$204 \quad c_{p r}^{R} \quad$ unit processing cost of product $p$ at process $r$.

$205 c_{i z o j}^{H I} \quad$ unit transportation cost of any product from forest area indicated by $(i z o) \in I_{i z o}$ to mill $j$.

$206 c_{i j h}^{H M} \quad$ unit transportation cost of any product between mill $i$ and $j$ by truck type $h$.

$207 c_{l h}^{M L} \quad$ unit transportation cost for backhaul route $l$ by truck type $h$. 
$208 a_{l f s o j}^{M L} \quad 1$; if backhaul trip $l$ picks up pile $f$ with sort group $S$ and owner $o$ at supply point $j$.

$209 b_{l f s o j}^{M L} \quad 1$; if backhaul trip $l$ delivers pile $f$ with sort group $S$ and owner ${ }_{o}$ to demand point $j$.

$210 \quad u_{p r q}^{R} \quad$ yield of process $r$ in producing product $q$ while it is fed by product $p$.

$211 k_{r}^{R+} \quad$ max capacity of process $r$.

$212 \quad k_{r}^{R-} \quad$ min capacity of process $r$.

$213 \quad k_{j h}^{H} \quad$ transportation capacity of truck type $h$ for mill $j$.

$214 \quad M \quad$ a big value.

215 To avoid nonlinearity in modelling we assume the proportion of available products in each pile (

$\left.216 a_{f l}^{F S}\right)$ is known. This value is estimated by average on proportion of available forest resources.

217 The decision variables used in the models are:

$218 y_{i}^{E} \quad 1$; if biorefinery $i$ is open, and 0 otherwise.

$219 \quad y_{i}^{T} \quad 1$; if terminal $i$ is open, and 0 otherwise.

$220 x_{i z o j}^{A} 1$; if forest block indicated by $(i z o) \in I_{i z o}$ is assigned to mill $j$, and 0 otherwise.

$221 x_{\text {sizo }}^{A P}$ percentage of harvesting in sort group $S$ from forest block indicated by $(i z o) \in I_{i z o}$.

$222 x_{f s c i z o j h}^{F F}$ flow of pile $f$ with sort group $S$ and species $c$ from forest block indicated by $(i z O) \in I_{i z o}$

223 to mill $j$ by truck type $h$.

$224 x_{f s c o i j h}^{M F}$ flow of pile $f$ with sort group $S$ and species ${ }_{c}$ belong to owner ${ }_{o}$ between mills $i$ and $j$

$225 \quad$ by truck type $h$.

$226 x_{l h}^{M L} \quad$ backhaul flow $l$ done by truck type $h$. 
$227 \quad x_{j p}^{E} \quad$ volume of final product $p$ produced at mill $j$.

$228 x_{f s o j}^{S} \quad$ sorting amount of pile $f$ with sort group $S$ and wood owner $o$ at mill $j$.

$229 x_{p s o r j}^{R}$ volume of inputted product $p$ with sorting group $S$ and wood owner $o$ to process $r$ at mill $230 j$.

231 Most of the decision variables are anticipation variables which are used to evaluate the strategic 232 decisions. As such, these anticipation decisions are not be operated. Instead, shorter-term tactical 233 and operational models will be used to find such operational decisions. Besides, the available 234 data is based on long-term allowable annual cut (AAC) models which essentially describe the 235 available volume per year. As these numbers are the same; it is possible to use only one time 236 period to make strategic decisions. Hence, we do not consider time horizon dimension for 237 decision variables.

238 The objective function is to maximize the profit (revenue from sales minus costs). The sales 239 value of final products is the revenue of the network. The included costs in the model are 240 harvesting, transportation, sorting, operating, and fixed cost to open new terminals and 241 biorefinery.

$$
\operatorname{Max} Z: \sum_{p \in P^{F}} \sum_{j \in N}\left(v_{p j}^{F} x_{j p}^{E}\right)-\sum_{s \in S} \sum_{(i z o) \in I_{i z o}} \sum_{p \in P^{R}}\left(c_{s}^{I} k_{i z o p} x_{s i z o}^{A P}\right)-\sum_{(i z o) \in I_{I z o}} \sum_{(f o j) \in E_{f o j}^{F F}} \sum_{s \in S} \sum_{c \in F^{C}} \sum_{h \in H}\left(c_{i z o j}^{H I} x_{f s c i z o j h}^{F F}\right)-
$$

$$
\begin{aligned}
& \sum_{(f o i j) \in E_{f o j}^{F M}} \sum_{s \in S} \sum_{c \in F^{C}} \sum_{h \in H}\left(c_{i j h}^{H M} x_{f s c o i j h}^{M F}\right)-\sum_{l \in L^{B}} \sum_{h \in H}\left(c_{l h}^{M L} x_{l h}^{M L}\right)-\sum_{f \in F^{p}} \sum_{s \in S} \sum_{o \in O} \sum_{j \in N}\left(c_{s j}^{S} x_{f s o j}^{S}\right)- \\
& \sum_{(p r) \in P_{p r}^{R+}} \sum_{s \in S} \sum_{o \in O} \sum_{(r j) \in P_{r j}^{R}}\left(c_{p r}^{R} x_{p s o r j}^{R}\right)-\sum_{i \in L^{T}} f_{i} y_{i}^{T}-\sum_{i \in L^{E}} f_{i} y_{i}^{E}
\end{aligned}
$$

243 The model is subjected to a set of constraints for capacity constraint, fibre delivery constraints, 244 balance constraints, flow constraints and demand constraints.

\section{Capacity constraints:}


246 The first set of constraints, harvest capacity constraint, assures the sum of a percentage of

247 harvested products in different sorting groups for each forest block should be less than one.

$248 \sum_{s \in S} x_{s i z o}^{A P} \leq 1$

$$
\forall(i, z, o) \in I_{i z o}
$$

249 The resource should satisfy mill demand; however each forest area may only be assigned to one 250 mill (constraint set (3)).

$251 \sum_{j \in N} x_{i z o j}^{A} \leq 1$

$$
\forall(i z o) \in I_{i z o}
$$

252 Constraint set (4) assures that only assigned forest area will be harvested.

$253 \sum_{s \in S} \sum_{z \in Z \mid(i z o) \in I_{i z o}} x_{s i z o}^{A P} \leq \sum_{j \in N} x_{i o j}^{A}$

$$
\forall i \in D, o \in O
$$

254 Constraint set (5) considers the restricted capacity of transportation of all transported products to 255 or from a mill.

256

$$
\begin{aligned}
& \sum_{(f o j) \in E_{f o j}^{F F}} \sum_{s \in S} \sum_{c \in F^{C}} \sum_{(i z o) \in I_{i z o}} x_{f s c i z o j h}^{F F}+\sum_{(f o i j) \in E_{f o j}^{M F}} \sum_{s \in S} \sum_{c \in F^{C}} x_{f s c o i j h}^{M F}+\sum_{l \in L^{B}} \sum_{(f o i j) \in E_{f o i j}^{M F}} \sum_{s \in S} b_{l f s o j}^{M L} x_{l h}^{M L}+{ }_{\forall j \in N, h \in H} \\
& \sum_{s \in S} \sum_{i \in N} \sum_{f \in P^{F}} \sum_{(f o j i) \in E_{f o j i}^{F M}} \sum_{c \in C} x_{f s c o j i h}^{M F}+\sum_{l \in L^{B}} \sum_{i \in N} \sum_{(f o j i) \in E_{f o j i}^{M F}} \sum_{s \in S} a_{l f s o j}^{M L} x_{l h}^{M L} \leq k_{h j}^{H}
\end{aligned}
$$

\section{Flow constraints}

258 The flow of wood in the network will start from the forest; therefore, all harvested resources

259 should be directly forwarded from the forest to mills (constraint set (6)).

260

$$
\sum_{p \in P^{R}} k_{i z o p} x_{s i z o}^{A P}-\sum_{(f o j) \in E_{f o j}^{F F}} \sum_{c \in F^{C}} \sum_{(o j h) \in H_{o j h}} x_{f s c i z o j h}^{F F}=0
$$$$
\forall(i z o) \in I_{i z o}, s \in S
$$

261 Moreover, constraint set (7) permits all the decision variables related to the biorefinery to have

262 the value 1 if the facilities are open. The same constraint exists for terminals. 
$\sum_{(f o j) \in E_{f o j}^{F F}} \sum_{(i z o) \in I_{i z o}} \sum_{s \in S} \sum_{c \in F^{C}} \sum_{(o j h) \in H_{o j h}} x_{f s c i z o j h}^{F F}+\sum_{(f o i j) \in E_{f o j}^{F M}} \sum_{s \in S} \sum_{c \in F^{C}} \sum_{(o j h) \in H_{o j h}} x_{f s c o i j h}^{M F}+$

263

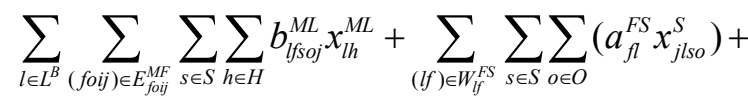

$\forall j \in l^{E} \bigcup l^{T}$

$\sum_{(r j) \in P_{r j}^{R}} \sum_{(f r) \in P_{r r}^{R-}} \sum_{(p r) \in P_{p r}^{R+}} \sum_{s \in S} \sum_{o \in O}\left(u_{p r f}^{R} x_{p s o r j}^{R}\right)+\sum_{(f o j i) \in E_{f o j i}^{F M}} \sum_{s \in S} \sum_{c \in C} \sum_{(o i h) \in H_{o i h}} x_{f s c o j i h}^{M F}+$

$\sum_{l \in L^{B}} \sum_{(f o j i) \in E_{f o j i}^{M F}} \sum_{s \in S} \sum_{h \in H} a_{l f s o j}^{M L} x_{l h}^{M L}+\sum_{f \in F^{P}} \sum_{s \in S} \sum_{o \in O} x_{j f s o}^{S}+\sum_{(p r) \in P_{p r}^{R+}} \sum_{s \in S} \sum_{(r j) \in P_{r j}^{R}} \sum_{o \in O} x_{p s o r j}^{R} \leq M y_{j}^{E}$

Balance constraints:

The balance constraints are defined to assure the equilibrium of inputs and outputs of each mill

for different sorting piles. Constraint set (8) assures the balance of raw material and intermediate

products in the format of different piles at mills. The input flow can be a flow from forest or

balance constraint for the intermediate product.

$\sum_{(f o j) \in E_{f o j}^{F F}} \sum_{c \in C} \sum_{(i z o) \in I_{i z o}} \sum_{(o j h) \in H_{o j h}} x_{f s c i z o j h}^{F F}+\sum_{(f o i j) \in E_{f o j i}^{F M}} \sum \sum_{c \in C} \sum_{(o j h) \in H_{o j h}} x_{f s c o i j h}^{M F}+$

$$
\begin{array}{ll}
\sum_{l \in L^{B}} \sum_{(f o i j) \in E_{o f j}^{F F}} \sum_{h \in H} b_{l f s o j}^{M L} x_{l h}^{M L}+\sum_{(l f) \in W_{l f}^{F S}} \sum_{o \in O}\left(a_{f l}^{F S} x_{j l s o}^{S}\right)+ & \forall j \in N, f \in F^{P}, \\
\sum_{(p r) \in P_{p r+}^{R+} \&(f r) \in P_{f-}^{R-}} \sum_{o \in O} \sum_{(r j) \in P_{r j}^{R}}\left(u_{p r f}^{R} x_{p s o r j}^{R}\right)-\sum_{(f o j i) \in E_{f o j}^{M F}} \sum_{c \in C} \sum_{(o i h) \in H_{o i h}} x_{f s c o j i h}^{M F}- & s \in S \\
\sum_{l \in L^{B}} \sum_{(f o j i) \in E_{f o j i}^{M F}} \sum_{h \in H} a_{l l s o i}^{M L} x_{l h}^{M L}-\sum_{o \in O} x_{j f s o}^{S}-\sum_{(f r) \in P_{f r}^{R+}} \sum_{o \in O} \sum_{(r j) \in P_{r j}^{R}} x_{f s o r j}^{R}=0 &
\end{array}
$$

271 The balance constraint for final product at mill is formulated in constraint set (9).

272

$\sum_{(f r) \in P_{f r}^{R+} \&(p r) \in P_{p r}^{R-}} \sum_{(j r) \in P_{j r}^{R}} \sum_{s \in S} \sum_{o \in O}\left(u_{f r p}^{R} x_{f s o j r}^{R}\right)-x_{j p}^{E}=0$

$$
\forall j \in N, p \in P^{F}
$$

Demand constraints

274 In our modelling, each process has a minimum and maximum production range capacity 275 (constraint (10)). For the energy plants, this constraint depends on the existence of them in our network structure (constraint 11). 
$\begin{array}{lll}277 & k_{r}^{R+} \leq \sum_{(f r) \in P_{f r}^{R+}} \sum_{s \in S} \sum_{o \in O} x_{f s o r j}^{R} \leq k_{r}^{R-} & \forall(r j) \in P_{r j}^{R} \\ 278 & k_{r}^{R+} y_{j}^{E} \leq \sum_{f \in F^{P} \mid(f r) \in P_{f r}^{R+}} \sum_{s \in S} \sum_{o \in O} x_{f s o j r}^{R} \leq k_{r}^{R+} y_{j}^{E} & \forall r \in R, j \in L^{E} \mid(r j) \in P_{r j}^{R}\end{array}$

\section{Variable definition constraints}

280 Finally, variables are defined in constraint set (12) and (13).

$281 y_{i}^{E}, y_{i}^{T}, x_{i z o j}^{A} \in\{0,1\}, x_{\text {sizo }}^{A P} \in\{0,1\}$

$282 \quad x_{f s c i z o j h}^{F F}, x_{f s c o i j h}^{M F}, x_{l h}^{M L}, x_{j p}^{E}, x_{j f s o}^{S}, x_{f s o r j}^{R} \geq 0$

283 CASE STUdY

284 Newfoundland is the insular portion of the Canadian province of Newfoundland and Labrador 285 situated in the North Atlantic Ocean. It has two million ha of productive forestland, within 18 286 districts, available or partially available for harvest. The lands are $96 \%$ owned by the public and $2874 \%$ by the private sector. The primary commercial species are black spruce (Picea mariana) and 288 balsam fir (Abies balsamea). There are four stakeholders in operation: Corner Brook Pulp and 289 Paper Limited (capacity 700,000 m³/yr), Burton's Cove Lumber Limited (capacity 70,000 $290 \mathrm{~m}^{3} / \mathrm{yr}$ ), Cottle's Island Lumber (capacity 40,000 m³/yr), and Sexton Lumber (capacity 180,000 $291 \mathrm{~m}^{3} / \mathrm{yr}$ ) respectively from west to east. The final products of the mills are mainly conventional 292 forest products, including lumber, pulp and paper, and energy. Corner Brook Pulp and Paper 293 holds timber tenure of $29 \%$ of the total public forestlands that has been called Kruger tenure. 294 This means other stakeholders are not permitted to harvest Kruger tenure (Sustainable Forest 295 Managment 2013). They have annual agreements with the government that allow them to harvest 296 specific areas.

297 A Newfoundland forest map is depicted in Figure 2. The green blocks in the picture are harvest 
298 areas of $2 \mathrm{~km} \times 2 \mathrm{~km}$ size. Newfoundland has 5,888 of such blocks for each, district number, 299 location, timber tenure holder, harvest capacity and fibre attributes are known by the 300 Newfoundland Centre for Forest Science and Innovation. However, such detailed information is 301 not necessary for a strategic tactical planning problem. Hence, we aggregate forest blocks to a 302 larger size called zone as a forest management unit. The same information for blocks is available 303 for each of 520 zones. Afterwards, we identify each harvest area with its district number, zone 304 number and tenure holder.

305 The transportation cost between all network nodes is calculated by FPInterface ${ }^{\mathrm{TM}}$. Information 306 comprising supply, harvesting and purchasing cost, yield of process and operation cost is 307 collected from each stakeholder. All values (revenues) and costs are evaluated as sold at mill 308 gate.

309 Fibre wood availability is one of the most challenging problems in this supply chain, particularly 310 for sawmills with their restricted timber tenure. Two reasons are the cause of this problem. First, 311 Newfoundland has a very acid soil with low fertility, which means large and vast supply areas. 312 Second, mills do not receive the quantity and quality of supply they require. Sorting yards with 313 the goal of delivering the right fibre to the right mill at the right time could significantly improve 314 current situation.

315 On the other hand, high transportation costs as well as environmental concerns are two other 316 significant problems for Newfoundland forest supply chain. The large resource distribution 317 increases the transportation distance. The west of the insular area is covered by balsam fir which 318 is valuable feedstock for Corner Brook Pulp and Paper; and conversely, the eastern part of the 319 island is covered by black spruce, a valuable raw material for sawmills. Moreover, transportation 320 in Newfoundland is operated by several independent organizations under different agreements. 
321 As a consequence, the supply delivery to Corner Brook Pulp and Paper is restricted to drivers

322 from a particular organization. This condition severely limits opportunity for collaboration in the

323 form of backhauling transportation.

324 Backhaul transportation could be a beneficial option to demonstrate the impact of collaboration, 325 to reduce transportation costs and greenhouse gas (GHG) emissions. We have designed three sets 326 of backhaul routes depicted in Figure 3. Each backhaul route characterizes the supply and 327 delivery nodes as well as the hauled products and their owner. The total saving in backhaul 328 transportation declared in the figure is calculated by the reduction in empty driving distances. 329 Moreover, we calculate the reduction in GHG emissions by using backhaul transportation in 330 equation (14) (Lakshmanan and Han 1997). The equation requires distance travel $\left(m^{3} \times k m\right)$, fuel 331 consumption $\left(\frac{\text { gallon }}{(100 \mathrm{~km})\left(\mathrm{m}^{3}\right)}\right)$; and fuel specific factor $\left(8.887 \times 10^{-3} \frac{\text { metrictons }}{\text { gallon }}\right)$ to calculate metric 332 tons of $\mathrm{CO} 2$ emissions.

$333 G H G=$ Distance travel $\times$ fuel consumption $\times$ fuel specific factor

334 The Newfoundland stakeholders are always searching for new products, processes, and markets 335 to increase their profit margin. Investigation of their current state and the availability of wood 336 energy in the forest have identified that new biorefinery are ideal options for improving their 337 profitability (Melendez Esquivel 2015). The new process could be installed either in green field 338 site or existing mills.

339 The case study in Newfoundland is a very good test for our proposed model. Twenty potential 340 locations for terminals and one potential location for a biorefinery are considered (Figure 4). 341 These locations represent large crossings in the road network and are identified together with 342 government representatives in collaboration with the stakeholders. Moreover, to evaluate the 
343 effect of each of the aforementioned actions we develop different scenarios. Each scenario is 344 optimized by the model and analyzed.

345 The scenarios are generated through a combination of assumptions. The assumptions are: 1)

346 allowable wood allocation in forest can be shared between all stakeholders; 2) accessibility of 347 sawmills to supply in the Kruger tenure; 3) possibility of using backhaul availability; 4) 348 terminals can be used; 5) biorefineries can be used. Assumption (1) gives the model the 349 possibility to allocate a harvest zone to more than one stakeholder. It is provided to model by 350 relaxing constraint set (3). Assumption (2) allows sawmills to harvest and transport from forest 351 zones located in the Kruger tenure to mills. Modification in the matrix of flows permits such 352 wood flows. Backhaul availability, terminals and a pellet mill are applied to model by their 353 decision variable whether they are zero or not. It is worth mentioning that assumption (1) - (3) 354 could be used directly if the stockholders accept collaboration. Hence, any scenario that includes 355 these assumptions represents collaboration concept.

356 The matrix of the scenarios is provided in Table 1. Scenario (1) presents the current situation of 357 the network where none of the aforementioned assumptions exists. The other scenarios, however, 358 are current situations considering one or several assumptions. For instance, in scenario 18, we 359 allow the optimization model to allocate a forest zone to different stakeholders, and to have 360 terminal opening opportunity. The other scenarios are interpreted in the same way considering its 361 active assumptions.

362 RESULTS

363 The model is implemented in the modelling language AMPL using CPLEX 12.0 as a solver. For 364 all numerical tests, we have used a standard PC with a 2.6 GHz processor and 42.0 GB RAM. 365 The size of each scenario, its solution time and its gap using CPLEX directly are presented in 
366

367

368

369

370

371

372

373

374

375

376

377

378

379

380

381

382

383

384

385

386

387

388

Table 2. The number of binary and linear variables as well as constraints is provided for each scenario. A restricted solution time of 1,800 seconds is considered for each problem. The gap determines the difference between best integer solution and best bound (from the LP relaxation) divided by the best bound. All problems were solvable with a maximum gap of $0.5 \%$.

For each scenario, we report on a set of key performance indicators (KPI). These KPIs, defined in Table 3, demonstrate how effectively each mill achieves the business objective and have been identified together with the stakeholders. They are reported for each stakeholder and for each scenario. KPIs RevInt and RevFin calculate respectively the revenue from selling Intermediate and final products at mill gates. KPIs 3 to 9 demonstrate strategic and tactical costs. The profit and total delivered product volume to each mill are calculated by KPIs TotalProfit and TotalIn, respectively. The KPI Profit/In represents the profit per one $\mathrm{m}^{3}$ of the proceeded supply. Finally, KPI GHG indicates the saving in GHG emissions using backhauling.

Table 4 reports total revenue of selling products, fixed cost, tactical cost and profit in millions of CAD dollars. Moreover, it reports total volume incoming product in million cubic metres. The profit per one $\mathrm{m}^{3}$ of incoming product as a key comparator of the scenarios is calculated in Profit/In column. The GHG column represents reduction of GHG emissions of all stakeholders. The model provides the detailed tactical information including the flow of products, the amount of sorted and operated product and the amount of backhauling for each stakeholder. It is worth mentioning that, tactical cost is the sum of harvesting, purchasing, transporting, sorting and operating costs.

As the table shows, in all of the scenarios, the optimization model supplies the stakeholders up to their maximum requirements, hence the total revenue is almost equal for all scenarios. The revenue of stakeholders will increase in the scenarios which open a pellet mill. For instance, the 
389

390

391

392

393

394

395

396

397

398

399

400

401

402

403

404

405

406

407

408

409

410

411

total revenue of scenarios $s_{1}$ and $s_{18}$ has a difference of around $\$ 6.7$ million which is principally created by the opening of the pellet mill. On the other hand, the flow of products within the network will reduce in all of the scenarios compared to the base case and according to reduction in tactical costs. Harvesting cost primarily depends on two factors including the accessibility of sawmills to Kruger tenure and having a pellet mill. If the mill demands have not been satisfied by harvesting operation, they will purchase wood supply from other mills.

As regards value creation, the model suggests the sorting opportunity for all scenarios to create higher value from the fibre. In all scenarios, the solutions supply stakeholders up to their maximum volume requirement; however different total profits are reported. This shows two key points: the first is, even in the base case scenario, the supply chain has the ability to provide secure fibre for all stakeholders; second, with the same input and more collaboration all stakeholders could generate more profit. Revenue, cost and profit for all scenarios are compared in Figures 5, 6 and 7, respectively. Comparison of Profit/In for different scenarios in Figure 7 presents a potential saving of $23.06 \%$ for the entire supply chain which could be created only by collaboration between stakeholders.

The overall localization decisions of the model are to open one terminal in the west of Newfoundland or the one in the east central region (Figure 8). The terminal in the West will facilitate the transportation to Corner Brook Pulp and Paper by their hauling fleets, and from that terminal to all stakeholders, the possibility of backhauling by other drivers will be provided. Moreover, in all scenarios with the possibility of wood exchange, all harvested pulp logs will be delivered directly to the terminal and then to Corner Brook Pulp and Paper. The terminal located in East is preferable if sawmills have accessibility to Kruger tenures. In this way, the decision supporting tool suggests harvesting the centre of Newfoundland and delivering to terminals in 
412 the centre, and then distributing the products. The pellet mill is a profitable decision when

413 sawmills have access to Kruger tenure, otherwise the restriction on resources will prevent such

414 investment. The location of selected terminals is also in accordance with the expectations of all 415 stakeholders.

416 CONCLUding REMARKS

417 We have developed an MIP model to design a forest biomass value chain with special 418 consideration of fibre value creation. Our developed model covers important strategic and 419 tactical decisions at the same time. The strategic decisions include locating new terminals and a 420 pellet mill. The network flows, harvesting, purchasing, transporting and sorting costs are 421 considered in the model to support a sustainable strategic decision. The backhauling 422 transportation opportunity is considered to reduce cost and GHG emissions.

423 Our generic model was validated with a real case study in the context of the Newfoundland 424 forest industry and has provided guidelines for this supply chain to progress. Two special 425 conditions of the case study including truck hauling regulation and timber tenure holders have 426 considerable effect on profitability of the industry. Moreover, the significant effect of 427 collaboration on network profitability has been reported.

428 Our developed optimization model has the ability to consider a new bio-process in its modelling. 429 However, a comprehensive market study is required to design a good product-process portfolio. 430 A fact in such a new market is the uncertainty about availability of resources, performance of 431 new processes and the market of new products. In this regard, uncertainty consideration in such 432 areas could be very interesting to study and to compare with the current findings. Moreover in 433 the new designed value chain the individual stakeholder saves differently, hence a study on 434 sharing profit mechanisms could introduce an applicable future work. 


\section{ACKNOWLEDGMENTS}

436 The author would like to acknowledge FORAC research consortium (Université Laval) and

437 NSERC Strategic Network on Value Chain Optimization for supporting this research. Moreover, 438 special thanks to FPInnovations' Mr. Dave Lepage, and the Centre for Forest Science and 439 Innovation Newfoundland's Mr. Bill Dawson, for providing data to validate our developed 440 model. We also sincerely thank the co-operation of the stakeholders: Mr. David Chamberlain, 441 Corner Brook Pulp and Paper Ltd; Mr. Fred Osmond, Burton's Cove Ltd; Mrs. Lorie Philpott, 442 Cottle's Island Ltd; and Mr. Kevin Sexton and Mr. Neil Greening, Sexton Lumber Ltd. 


\section{REFERENCES}

ALAM, M. B., SHAHI, C. \& PULKKI, R. 2014. Economic impact of enhanced forest inventory information and merchandizing yards in the forest product industry supply chain. Socio-Economic Planning Sciences, 48, 189-197.

CAMBERO, C., SOWLATI, T., MARINESCU, M. \& RÖSER, D. 2014. Strategic optimization of forest residues to bioenergy and biofuel supply chain. International Journal of Energy Research, 39, 439-452.

CARLGREN, C. G., CARLSSON, D. \& RÖNNQVIST, M. 2006. Log sorting in forest harvest areas integrated with transportation planning using backhauling. Scandinavian Journal of Forest Research, 21, 260-271.

CARLSSON, D. \& RÖNNQVIST, M. 2005. Supply chain management in forestry-case studies at Södra Cell AB. European Journal of Operational Research, 163, 589616.

CARLSSON, D. \& RÖNNQVIST, M. 2007. Backhauling in forest transportation models, methods and practical usage. Canadian Journal of Forest Researh, 37, 2612-2613.

D’AMOURS, S., RÖNNQVIST, M. \& WEINTRAUB, A. 2008. Using Operational Research for supply chain planning in the forest product industry. INFOR, 46, 4764.

DANSEREAU, L. P., EL-HALWAGI, M. M. \& STUART, P. R. 2012. Value-Chain Planning in the Forest Biorefinery: Case Study Analyzing Manufacturing Flexibility. Journal of Science and Technology for Forest Products and Processes, 2, 60-69. 
DRAMM, J. R., GOVETTT, R., BILEK, T. \& JACKSON, G. L. 2004. Log sort yard economics, planning, and feasibility, Gen. Tech. Rep. FPL-GTR-146. Madison, WI: U.S. Department of Agriculture, Forest Service, Forest Products Laboratory.

EKŞIOĞLU, S. D., ACHARYA, A., LEIGHTLEY, L. E. \& ARORA, S. 2009. Analyzing the design and management of biomass-to-biorefinery supply chain. Computers \& Industrial Engineering, 57, 1342-1352.

FENG, Y., D’AMOURS, S., NOURELFATH, M. \& LEBEL, L. 2010. Integrated Forest Biorefinery Supply Chain Network Design Using Mathematical Programming Approach. In: STUART, P. R. \& EL-HALWAGI, M. M. (eds.) Integrated Biorefineries: Design, Analysis, and Optimization. CRC Press/Taylor \& Francis.

FORSBERG, M., FRISK, M. \& RÖNNQVISTY, M. 2005. FlowOpt - A Decision Support Tool for Strategic and Tactical Transportation Planning in Forestry. International Journal of Forest Engineering, 16, 101-114.

FROMBO, F., MINCIARDI, R., ROBBA, M., ROSSO, F. \& SACILE, R. 2009. Planning woody biomass logistics for energy production: A strategic decision model. Biomass and Bioenergy, 33, 372-383.

GUNNARSSON, H., RÖNNQVIST, M. \& LUNDGREN, J. T. 2004. Supply chain modelling of forest fuel. European Journal of Operational Research, 158, 103123.

KIM, J., REALFF, M. J., LEE, J. H., WHITTAKER, C. \& FURTNER, L. 2011. Design of biomass processing network for biofuel production using an MILP model. Biomass and Bioenergy, 35, 853-871. 
LAKSHMANAN, T. R. \& HAN, X. 1997. Factors underlying transportation CO2 emissions in the U.S.A.: A decomposition analysis. Transportation Research Part D: Transport and Environment, 2, 1-15.

LEHOUX, N., MARIER, P., D'AMOUR, S., OUELLET, D. \& BEAULIEU, J. 2012. The value creation network of Canadian wood fibre. In: CANADA, N. R. (ed.). Canadian Forest Service Publications: CIRRELT report, 210 p.

MELENDEZ ESQUIVEL, J.A. 2015. Biomass procurement cost minimization for implementation of a retrofit biorefinery in a pulp and paper mill. P.hD. thesis, Department of Chemical Engineering, École Polytechnique de Montréal, Montréal, Québec, Canada.

MOCKLER, P. \& FAIRBAIRN, J. 2009. The Canadian Forest Sector: Past, Present, Future. In: Forestry, A. A. (ed.), Intern report of the standing senate comittee on agricalture and forestry, $62 \mathrm{p}$.

MOCKLER, P. \& ROBICHAUD, F. 2011. the canadian forest sector: a future based on innovation. In: Forestry, A. A. (ed.), final report on standinge senate committee on agriculture and forestry, $184 \mathrm{p}$.

NATURAL RESOURCES OF CANADA (A). 2016. Industry transformation [Online]. Available from http://www.nrcan.gc.ca/forests/industry/overview/13311.

NATURAL RESOURCES OF CANADA (B). 2016. Statistical data [Online]. Available from http://cfs.nrcan.gc.ca/statsprofile.

PALANDER, T. \& VÄÄTÄINEN, J. 2005. Impacts of interenterprise collaboration and backhauling on wood procurement in Finland. Scandinavian Journal of Forest Research, 20, 177-183. 
SHABANI, N., AKHTARI, S. \& SOWLATI, T. 2013. Value chain optimization of forest biomass for bioenergy production: A review. Renewable and Sustainable Energy Reviews, 23, 299-311.

$\begin{array}{lll}\text { SUSTAINABLE } & \text { FOREST } & \text { MANAGMENT. }\end{array}$ http://www.sfmcanada.org/images/Publications/EN/NL_info_Provinces_and_terri tories_EN.pdf.

TRONCOSO, J., D’AMOURS, S., FLISBERG, P., RÖNNQVIST, M. \& WEINTRAUB, A. 2015. A mixed integer programming model to evaluate integrating strategies in the forest value chain — a case study in the Chilean forest industry. Canadian Journal of Forest Research, 45, 937-949.

WEINTRAUB, A. \& EPSTEIN, R. 2005. The Supply Chain in the Forest Industry: Models and Linkages. In: GEUNES, J., PARDALOS, P. M. \& ROMEIJN, H. E. (eds.) Supply Chain Management: Models, Applications, and Research Directions. Boston, MA: Springer US. 
Table 1. Developed scenarios based on the five defined assumptions

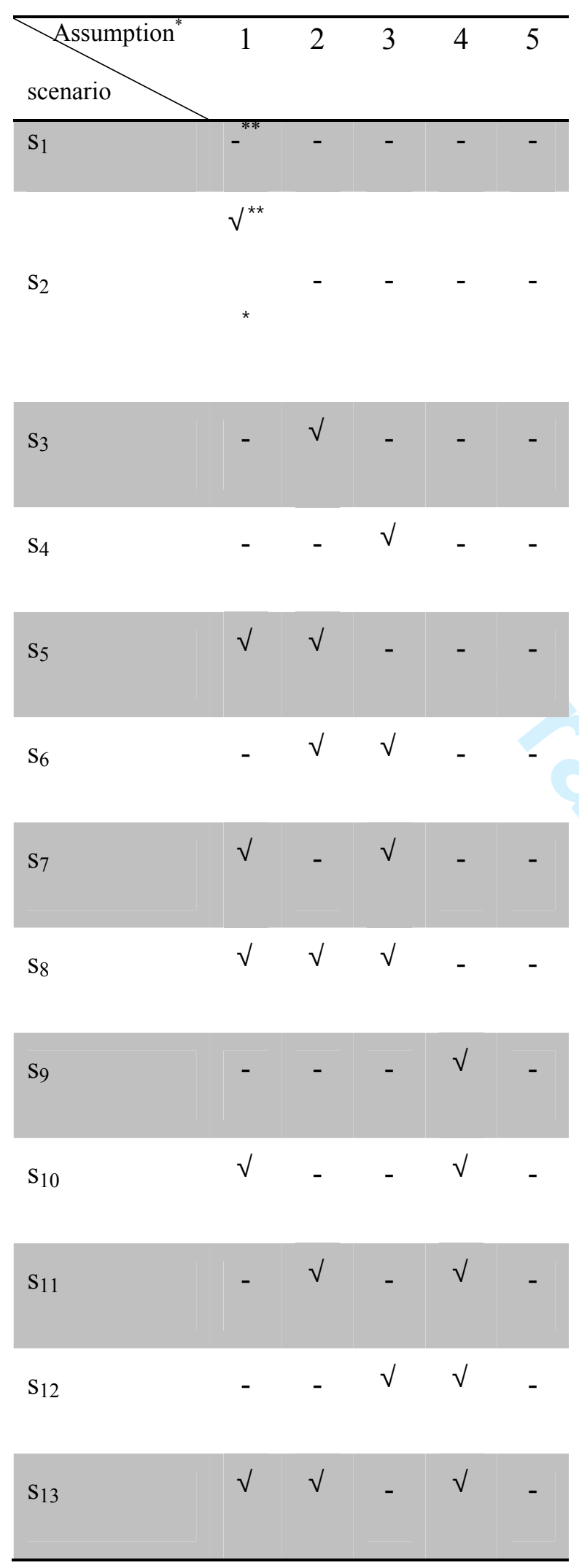




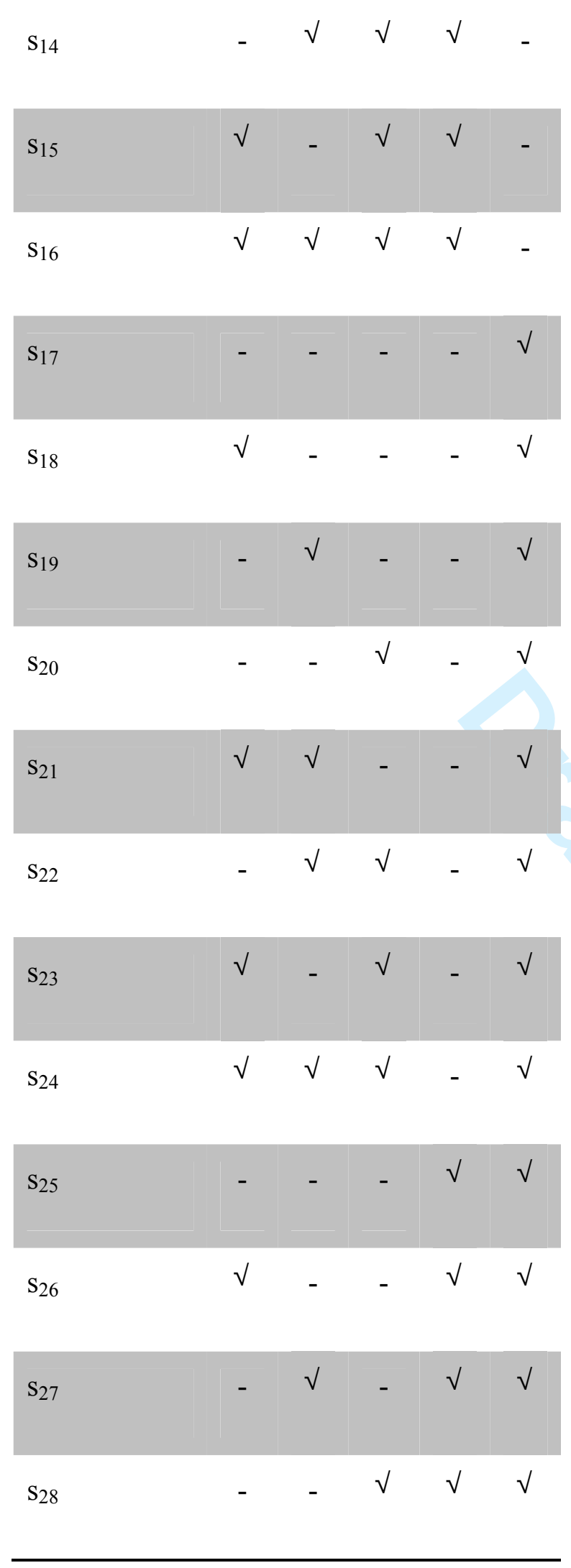

https://mc06.manuscriptcentral.com/cjfr-pubs 


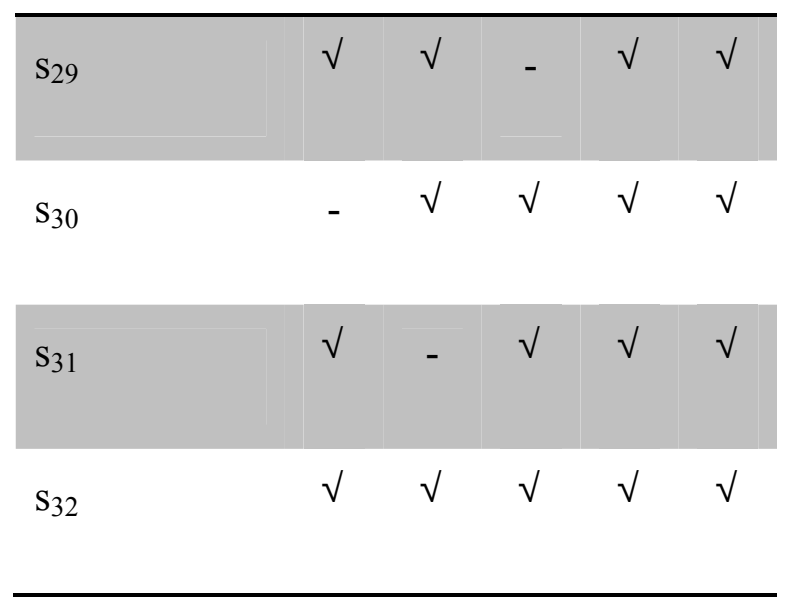

*Assumptions are as follows:

1) Allowable wood allocation in forest between all stakeholders; 2) accessibility of sawmills to Kruger tenure; 3) backhaul availability; 4) terminals opening; 5) biorefinery opening.

**Hyphen determines that assumption in the related scenario is inactive $* * *$ Check mark determines that assumption in the related scenario is active 
Table 2. Size of each scenario using CPLEX directly

\begin{tabular}{|c|c|c|c|c|c|}
\hline scenario & binary variables & linear variables & constraints & solution time(s) & gap(\%) \\
\hline $\mathrm{S}_{1}$ & 13,900 & 21,345 & 16,780 & 0.53 & 0.00 \\
\hline $\mathrm{S}_{2}$ & 1,520 & 21,345 & 3,205 & 0.22 & 0.00 \\
\hline $\mathrm{S}_{3}$ & 13,900 & 21,823 & 17,038 & 0.66 & 0.00 \\
\hline $\mathrm{S}_{4}$ & 13,900 & 21,385 & 16,788 & 0.52 & 0.02 \\
\hline $\mathrm{S}_{5}$ & 1,580 & 21,823 & 3,463 & 0.16 & 0.00 \\
\hline $\mathrm{S}_{6}$ & 13,900 & 21,930 & 17,054 & 0.94 & 0.04 \\
\hline $\mathrm{S}_{7}$ & 0 & 21,385 & 1,693 & 0.02 & 0.00 \\
\hline $\mathrm{S}_{8}$ & 21 & 176,036 & 7,355 & 21.40 & 0.00 \\
\hline $\mathrm{S}_{9}$ & 13,920 & 162,193 & 32,768 & 1800.08 & 0.13 \\
\hline $\mathrm{S}_{10}$ & 20 & 162,193 & 7,273 & 11.67 & 0.00 \\
\hline $\mathrm{S}_{11}$ & 13,920 & 162,811 & 32,846 & 1800.00 & 0.18 \\
\hline $\mathrm{S}_{12}$ & 13,920 & 167,349 & 32,772 & 132.38 & 0.11 \\
\hline $\mathrm{S}_{13}$ & 12,000 & 16,281 & 19,271 & 14.59 & 0.00 \\
\hline $\mathrm{S}_{14}$ & 13,920 & 171,843 & 32,854 & 221.05 & 0.12 \\
\hline $\mathrm{S}_{15}$ & 20 & 167,349 & 7,277 & 24.12 & 0.22 \\
\hline
\end{tabular}




\begin{tabular}{|c|c|c|c|c|c|}
\hline $\mathrm{S}_{16}$ & 20 & 171,843 & 7,299 & 1711 & 0.17 \\
\hline $\mathrm{S}_{17}$ & 13,901 & 31,410 & 21,822 & 1.4 & 0.00 \\
\hline $\mathrm{S}_{18}$ & 1 & 13,410 & 6,227 & 0.69 & 0.00 \\
\hline $\mathrm{S}_{19}$ & 13,901 & 32,028 & 22,106 & 1.36 & 0.79 \\
\hline $\mathrm{S}_{20}$ & 13,901 & 31,450 & 21,830 & 1.17 & 0.00 \\
\hline $\mathrm{S}_{21}$ & 2,101 & 32,028 & 8,531 & 1.39 & 0.00 \\
\hline $\mathrm{S}_{22}$ & 13,901 & 32,108 & 22,122 & 1.33 & 0.17 \\
\hline $\mathrm{S}_{23}$ & 1 & 31,450 & 6,235 & 1.48 & 0.00 \\
\hline $\mathrm{S}_{24}$ & 1 & 32,108 & 6,447 & 0.64 & 0.02 \\
\hline $\mathrm{S}_{25}$ & 13,921 & 166,246 & 33,318 & 1800.08 & 0.46 \\
\hline $\mathrm{S}_{26}$ & 21 & 166,246 & 7,323 & 17.25 & 0.00 \\
\hline $\mathrm{S}_{27}$ & 13,921 & 167,004 & 33,422 & 1801.11 & 0.16 \\
\hline $\mathrm{S}_{28}$ & 13,921 & 171,402 & 33,322 & 78.62 & 0.08 \\
\hline $\mathrm{S}_{29}$ & 12,521 & 167,004 & 19,847 & 17.04 & 0.00 \\
\hline $\mathrm{S}_{30}$ & 13,921 & 176,036 & 33,430 & 834.93 & 0.05 \\
\hline $\mathrm{S}_{31}$ & 21 & 171,402 & 7,327 & 35.04 & 0.00 \\
\hline $\mathrm{S}_{32}$ & 21 & 176,036 & 7,355 & 22.22 & 0.00 \\
\hline
\end{tabular}


Table 3. Key performance indicator definition

\begin{tabular}{|c|c|c|}
\hline Item & KPI & Description \\
\hline 1 & RevFin & Revenue from selling products \\
\hline 2 & RevInt & Revenue from selling products \\
\hline 3 & FixCost & Fixed cost to open terminals or pellet mill \\
\hline 4 & HvCost & Harvesting cost for each sort group \\
\hline 5 & PurCost & Purchasing cost for each sort group \\
\hline 6 & SortCost & Sorting cost for each sort group \\
\hline 7 & OperCost & Operating cost for each sort group \\
\hline 8 & TransCost & Transportation cost for each sort group \\
\hline 9 & TotalProfit & Total profit (revenue - costs) \\
\hline 10 & TotalIn & Total $\mathrm{m}^{3}$ input to mills \\
\hline 11 & Profit/In & Profit per $\mathrm{m}^{3}$ of input products at each mill \\
\hline 12 & GHG & Tonnes of GHG emissionss reduction by backhaul \\
\hline
\end{tabular}


Table 4. The optimization result for each of the scenarios (revenue, costs and profit are in million CAD)

\begin{tabular}{|c|c|c|c|c|c|c|c|}
\hline Scen & Total Revenue & FixCost & Tactical cost & TotalProfit & TotalIn & Profit/IN & GHG \\
\hline $\mathrm{S}_{1}$ & 197.32 & 0.00 & 129.02 & 68.30 & 1.45 & 47.09 & - \\
\hline $\mathrm{S}_{2}$ & 196.78 & 0.00 & 128.34 & 68.44 & 1.44 & 47.43 & - \\
\hline $\mathrm{S}_{3}$ & 195.36 & 0.00 & 126.71 & 68.64 & 1.45 & 47.29 & - \\
\hline $\mathrm{S}_{4}$ & 194.55 & 0.00 & 122.16 & 72.39 & 1.43 & 50.66 & 745.6 \\
\hline $\mathrm{S}_{5}$ & 189.23 & 0.00 & 117.12 & 72.11 & 1.26 & 57.46 & - \\
\hline $\mathrm{S}_{6}$ & 193.86 & 0.00 & 117.56 & 76.30 & 1.43 & 53.39 & 731.2 \\
\hline$S_{7}$ & 194.52 & 0.00 & 122.00 & 76.38 & 1.43 & 50.74 & 750.3 \\
\hline $\mathrm{S}_{8}$ & 191.04 & 0.00 & 114.92 & 76.12 & 1.31 & 57.95 & 498.3 \\
\hline $\mathrm{S}_{9}$ & 199.28 & 0.50 & 130.26 & 69.02 & 1.53 & 45.24 & - \\
\hline $\mathrm{S}_{10}$ & 195.67 & 0.50 & 123.75 & 71.92 & 1.42 & 50.55 & - \\
\hline $\mathrm{s}_{11}$ & 198.36 & 0.50 & 129.20 & 69.16 & 1.53 & 45.18 & - \\
\hline $\mathrm{s}_{12}$ & 194.55 & 0.00 & 122.16 & 72.39 & 1.43 & 50.66 & 745.6 \\
\hline $\mathrm{S}_{13}$ & 192.32 & 0.50 & 119.60 & 72.72 & 1.32 & 55.09 & - \\
\hline $\mathrm{S}_{14}$ & 193.86 & 0.00 & 117.56 & 76.30 & 1.43 & 53.39 & 731.2 \\
\hline $\mathrm{S}_{15}$ & 195.68 & 0.50 & 122.68 & 59.26 & 1.17 & 50.71 & 474.7 \\
\hline
\end{tabular}




\begin{tabular}{|c|c|c|c|c|c|c|c|}
\hline $\mathrm{s}_{16}$ & 190.97 & 0.00 & 114.86 & 76.12 & 1.31 & 57.95 & 498.3 \\
\hline $\mathrm{s}_{17}$ & 197.54 & 0.00 & 129.24 & 68.30 & 1.45 & 47.09 & - \\
\hline $\mathrm{S}_{18}$ & 203.99 & 1.00 & 132.71 & 71.28 & 1.45 & 49.28 & - \\
\hline $\mathrm{s}_{19}$ & 195.36 & 0.00 & 126.72 & 68.65 & 1.45 & 47.36 & - \\
\hline $\mathbf{S}_{20}$ & 194.55 & 0.00 & 122.16 & 72.39 & 1.43 & 50.66 & 745.6 \\
\hline $\mathbf{S}_{21}$ & 189.29 & 0.00 & 117.18 & 72.11 & 1.26 & 57.46 & - \\
\hline $\mathrm{S}_{22}$ & 194.06 & 0.00 & 117.76 & 76.30 & 1.43 & 53.39 & 731.2 \\
\hline $\mathrm{s}_{23}$ & 194.52 & 0.00 & 122.00 & 76.38 & 1.43 & 50.74 & 750.3 \\
\hline $\mathrm{S}_{24}$ & 190.95 & 0.00 & 114.83 & 76.12 & 1.31 & 57.95 & 498.3 \\
\hline $\mathrm{S}_{25}$ & 199.28 & 0.50 & 130.26 & 68.87 & 1.54 & 45.24 & - \\
\hline $\mathrm{S}_{26}$ & 195.79 & 0.50 & 123.87 & 71.92 & 1.42 & 50.55 & - \\
\hline $\mathbf{S}_{27}$ & 198.18 & 0.50 & 129.01 & 69.17 & 1.53 & 45.35 & - \\
\hline $\mathbf{S}_{28}$ & 194.55 & 0.00 & 122.16 & 72.39 & 1.43 & 50.66 & 745.6 \\
\hline $\mathrm{s}_{29}$ & 192.46 & 0.50 & 119.74 & 144.89 & 1.32 & 55.09 & - \\
\hline $\mathrm{S}_{30}$ & 193.92 & 0.00 & 117.63 & 76.30 & 1.43 & 53.40 & 731.2 \\
\hline $\mathrm{S}_{31}$ & 195.68 & 0.50 & 122.68 & 60.14 & 1.44 & 50.71 & 474.7 \\
\hline $\mathrm{S}_{32}$ & 191.04 & 0.00 & 114.92 & 76.12 & 1.31 & 57.95 & 498.3 \\
\hline
\end{tabular}




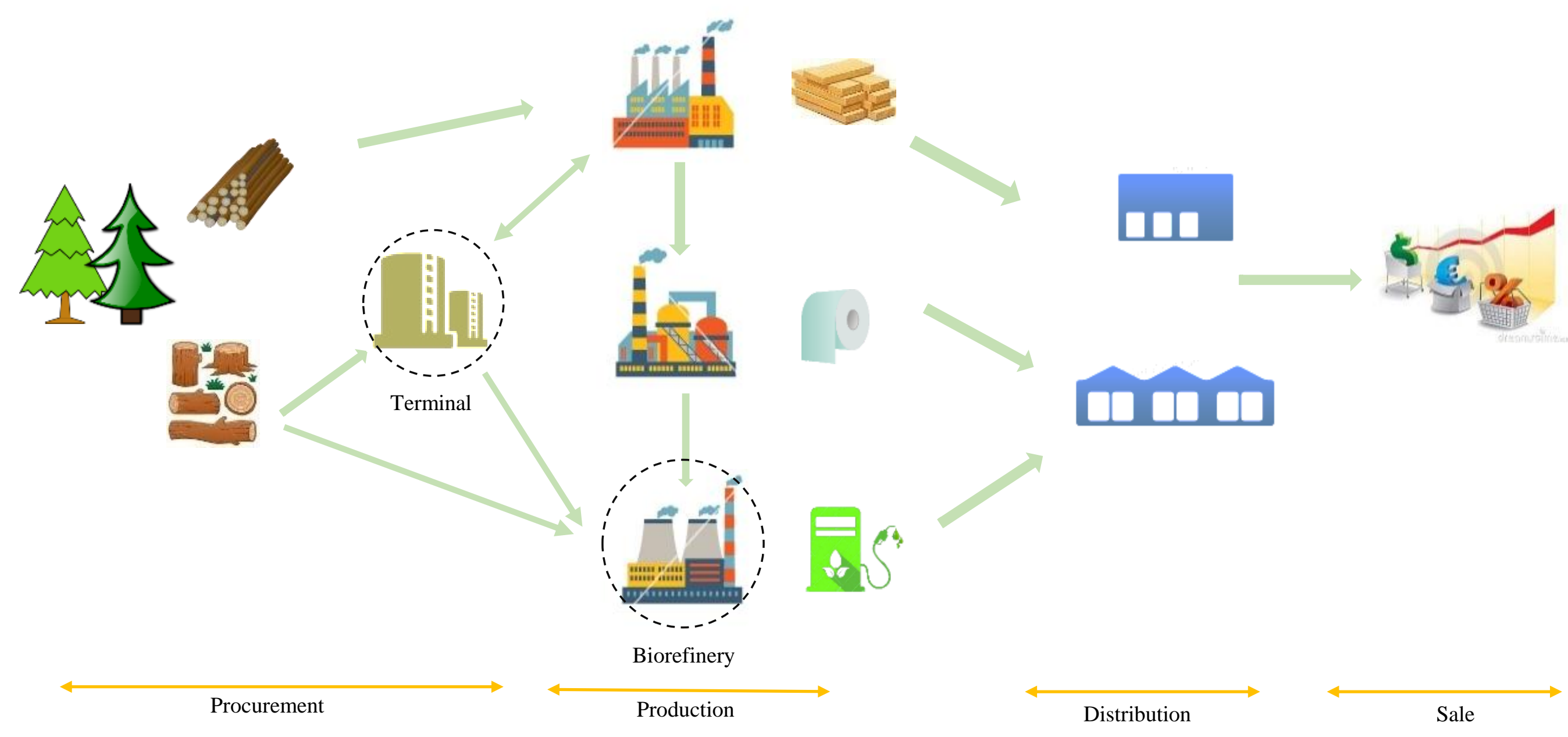




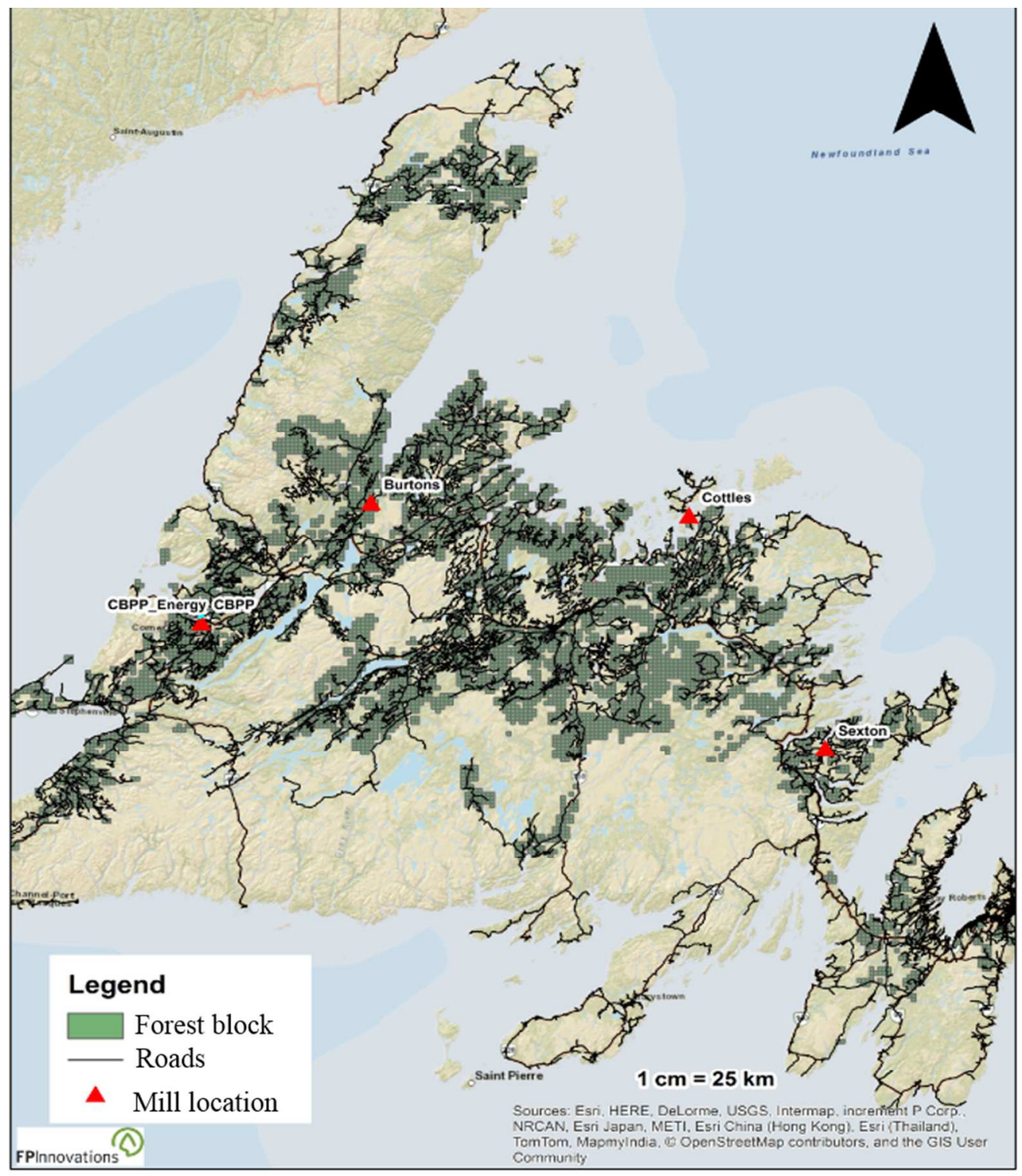

Figure 2. Map of Newfoundland forest industry 


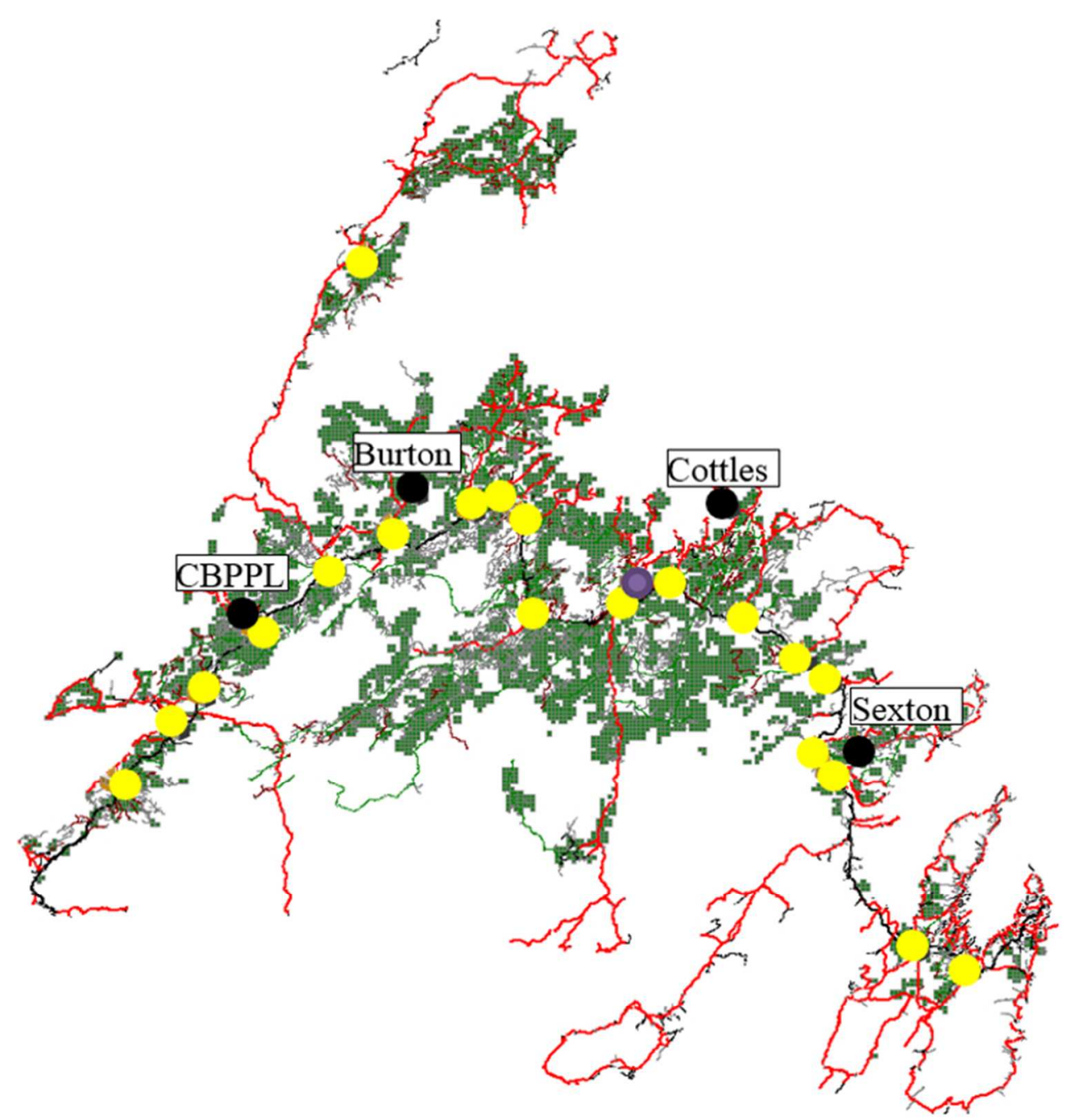

Figure 3. Potential location for terminals (yellow points) and pellet mill (purple point), the mills are depicted by black points (map is taken from FPInterfaceTM) 


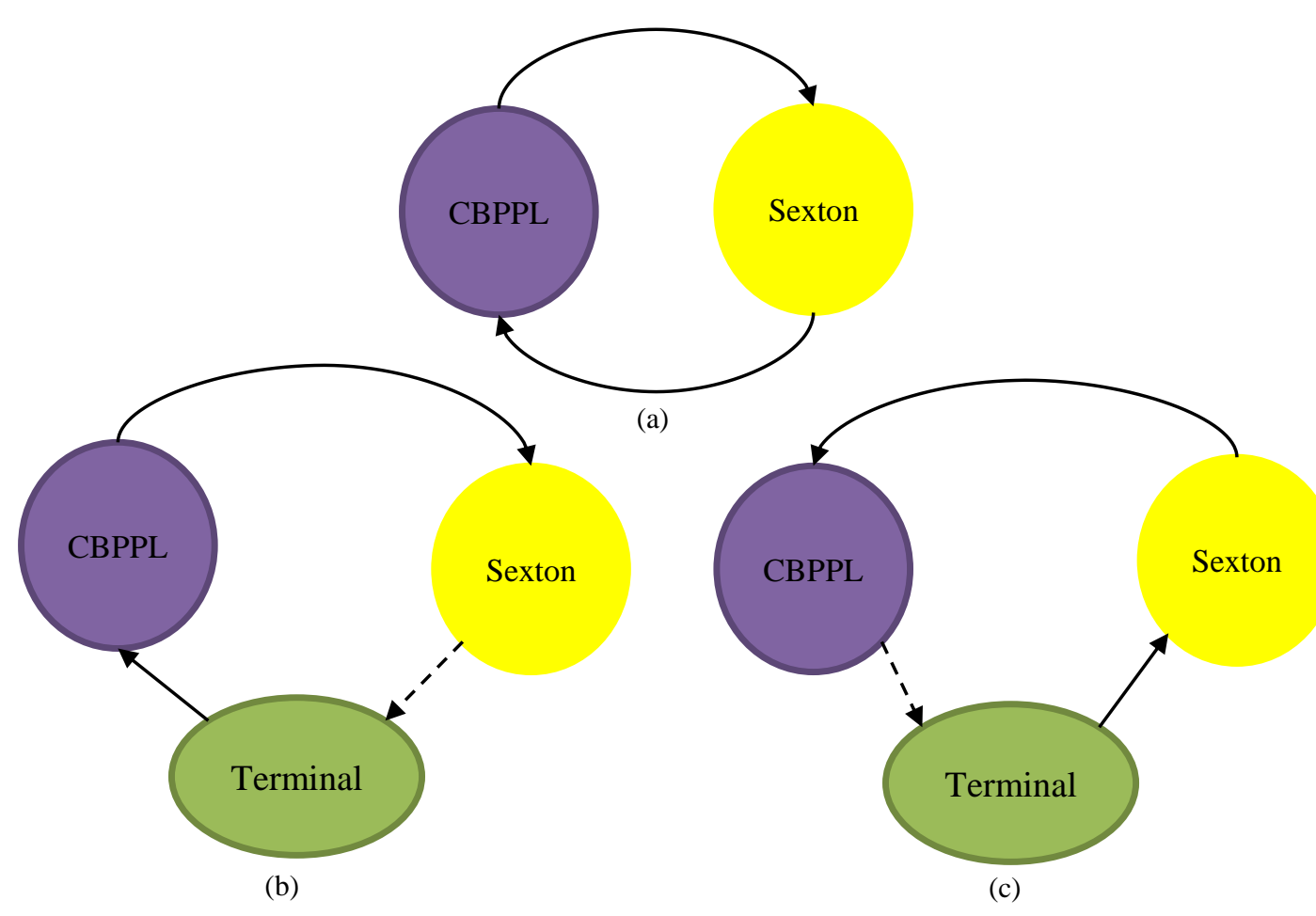

Figure 4. The possible backhaul routes: (a) Newfoundland's principle backhaul route without empty driving between Corner Brook Pulp and Paper (CBPPL) and Sexton Lumber. (b and c) Backhaul routes with more full load trips rather than empty driving between Corner Brock pulp and paper Ltd, Sexton Lumber and an open terminal. 


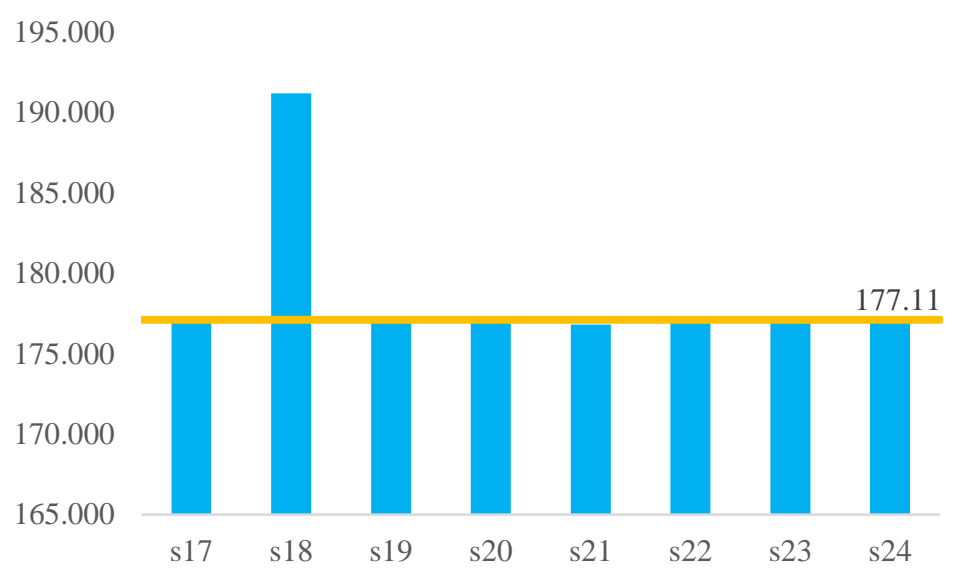

195.000

190.000 185.000

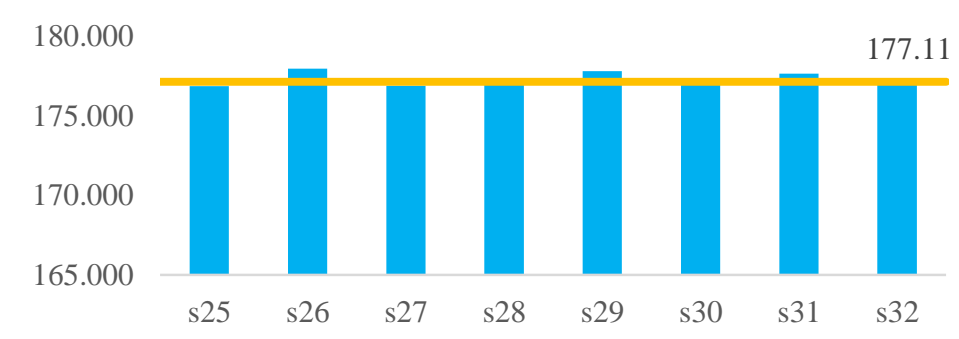

(c)

(d)

Rev reference line

Figure 5. Sum of revenue for selling products. The orange line in all figures is the total revenue for base case scenario. The scenarios are compared in four categories:

(a) scenarios without terminal and pellet mill opening assumptions, (b) scenarios with terminal opening assumption, (c) scenarios with pellet mill opening assumption, and (d) scenarios with terminal and pellet mill opening assumptions. 


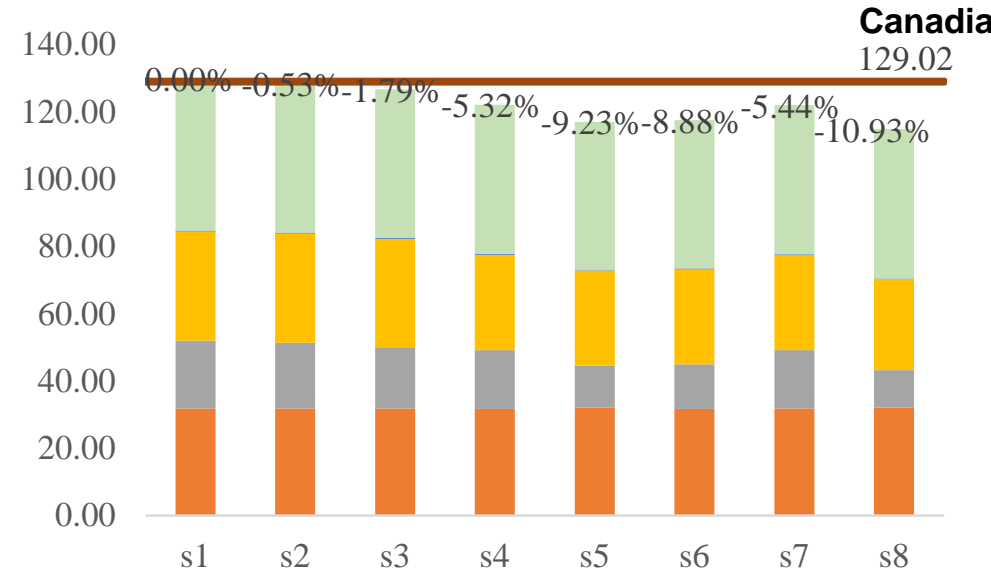

(a)

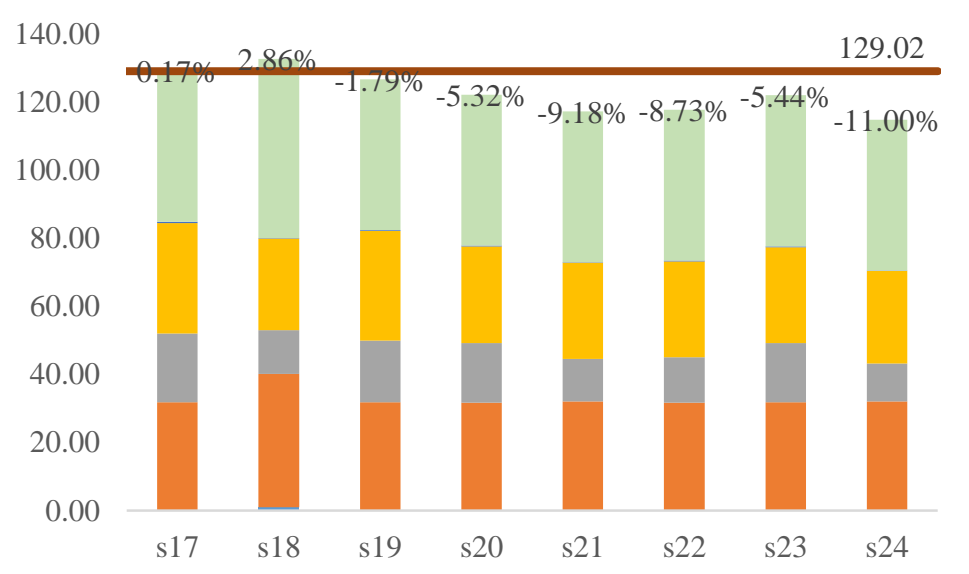

(c)

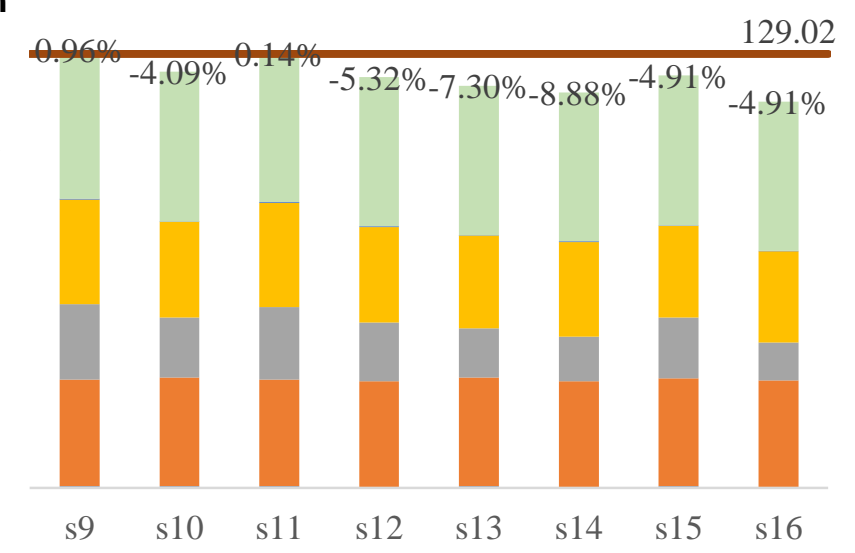

(b)

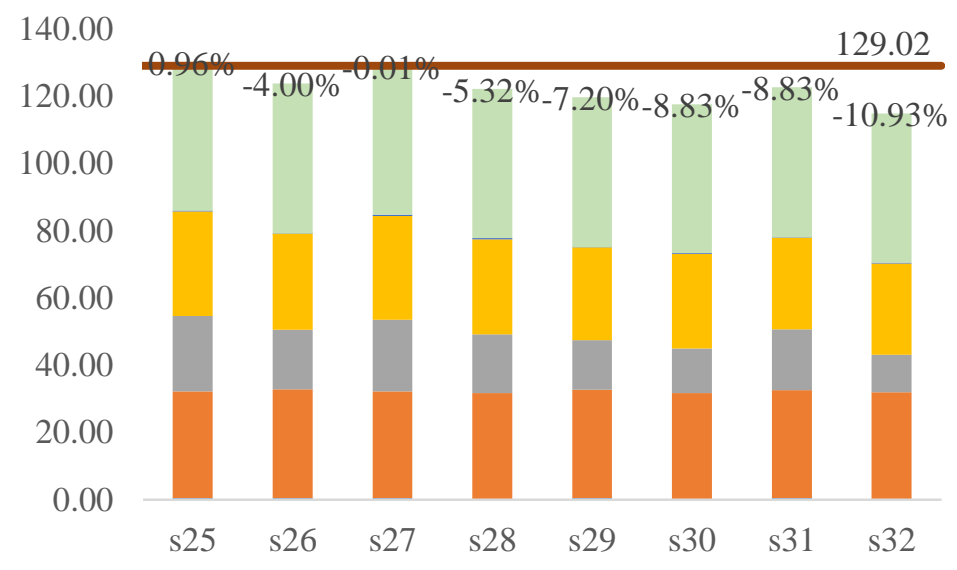

(d)

fixCost HvCost $\backsim$ PurCost TransCost $=$ SortCost $\quad$ OperCost refence Cost

Figure 6. Total network cost for each scenario. The brown line in all figures is total cost for base case scenario. The scenarios are compared in four categories: (a) scenarios without terminal and pellet mill opening assumptions, (b) scenarios with terminal opening assumption, (c)

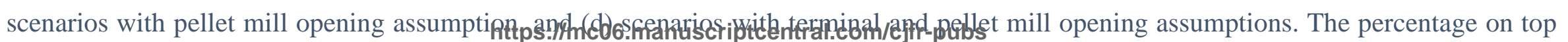




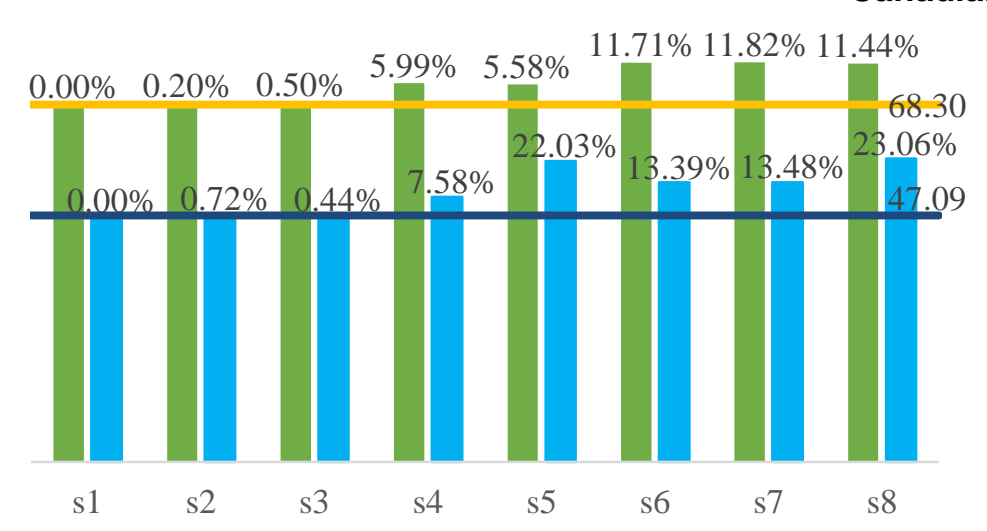

(a)

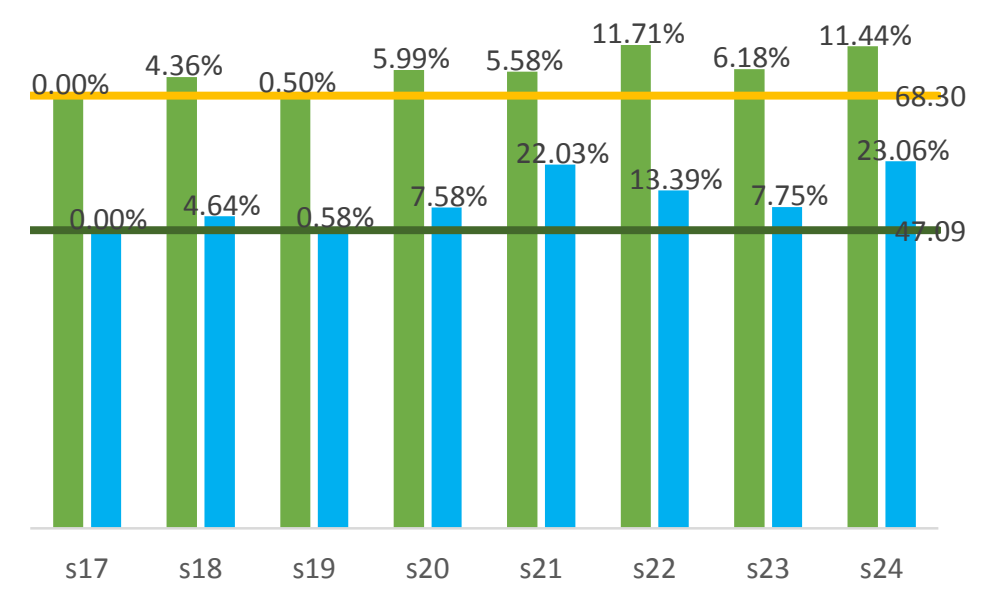

(c)

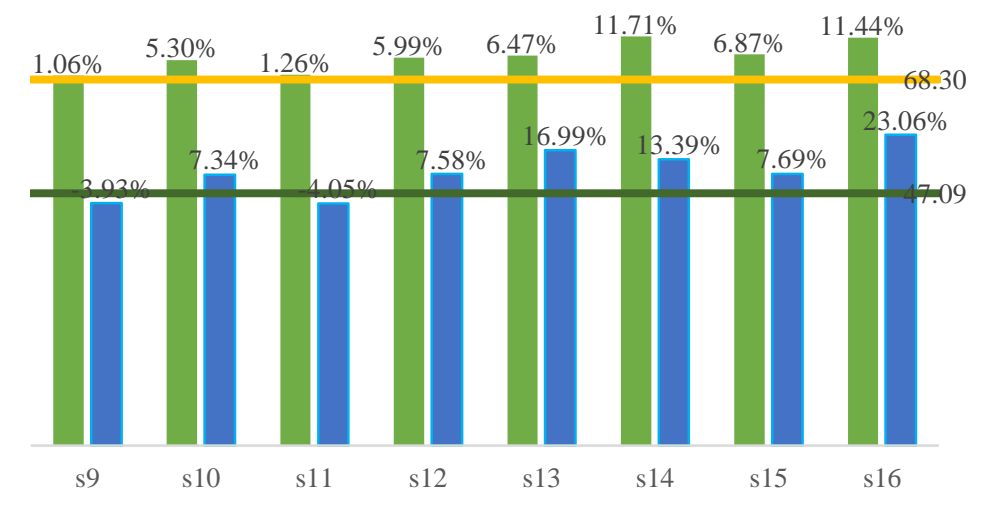

(b)

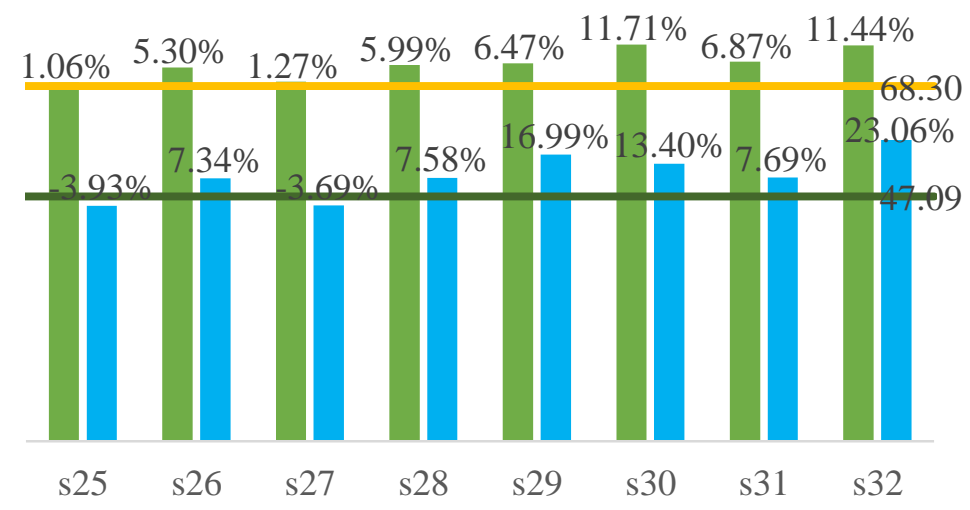

(d)

profit $\quad$ profit $/ \mathrm{m}^{3}$

reference profit

reference profit $/ \mathrm{m}^{3}$

Figure 7. Total network profit and profit $/ \mathrm{m} 3$ for each scenario. The orange and green lines in all figures are total profit and profit $/ \mathrm{m} 3$ for base case scenario. The scenarios are compared in four categories: (a) scenarios without terminal and pellet mill opening assumptions, (b) scenarios

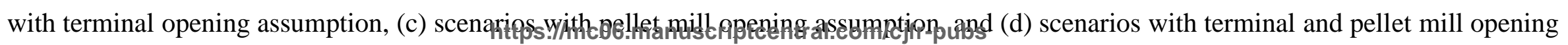
assumptions. The percentage on top of all bars determines the difference between the scenario and their corresponding reference line. 


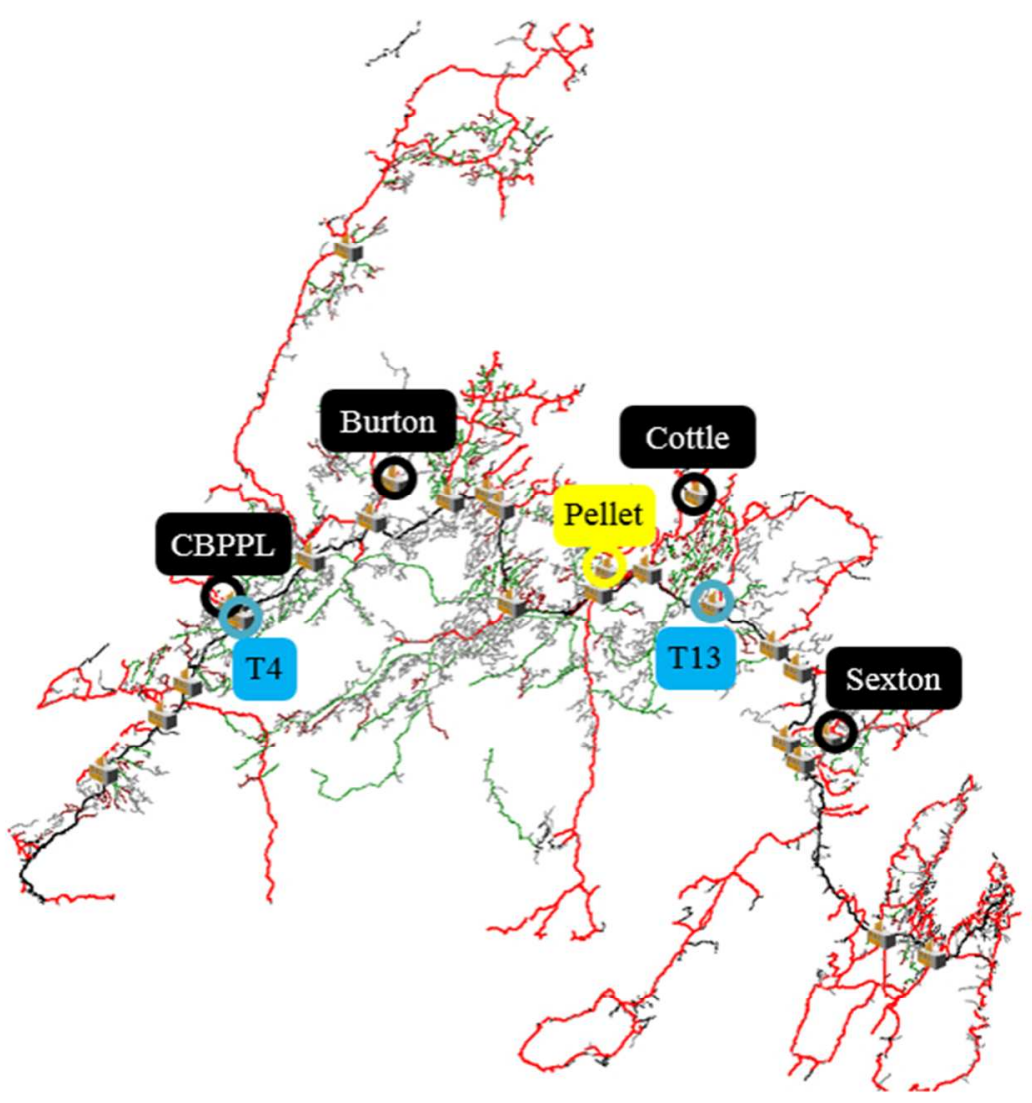

Figure 8. Selected facilities location in different scenarios (map is taken from FPInterfaceTM) 